\title{
Echinococcosis in children: Experience in a tertiary care hospital outside the endemic area
}

\author{
Griselda Berberian, M.D. ${ }^{a}$, M. Teresa Rosanova, M.D. ${ }^{a}$, Laura Inda, M.D. ${ }^{a}$, Claudia Sarkis, M.D. ${ }^{a}$, \\ Horacio Questa, M.D. ${ }^{b}$, Patricia Paulin, Biochemist ${ }^{c}$, Marina Costa, Biochemist ${ }^{d}$ and Moira Taicz, M.D. ${ }^{a}$
}

\begin{abstract}
Echinococcosis is a zoonotic disease that is widely spread across Argentina and worldwide. It is acquired during childhood but it is more common during adulthood. The pediatric population accounts for $10-20 \%$ of all cases.

This study included 47 children diagnosed with echinococcosis over a 20 -year period. Their median age was 8 years old (range: 3-17); most patients had some epidemiological history, such as having lived or living in an endemic or rural area and/ or having dogs that are fed with viscera. Findings included $85 \%$ of single cysts in only one parenchyma. Cysts were most commonly located in the liver and lungs. Medical/surgical treatment was carried out in 45 patients (96\%). Subsequent complications were observed in 13 patients $(28 \%)$, which were more common in those with lung cysts. Three patients $(6 \%)$ relapsed 1-24 months later and required a new surgery. No patient died as a result of the parasitic disease.

Key words: echinococcosis, child.
\end{abstract}

http:/ / dx.doi.org/10.5546/aap.2017.eng.282

To cite: Berberian G, Rosanova MT, Inda L, et al. Echinococcosis in children: Experience in a tertiary care hospital outside the endemic area. Arch Argent Pediatr 2017;115(3):282-286.

a. Department of Epidemiology and Infectious Diseases of Hospital de Pediatría "Prof. Dr. Juan P. Garrahan," Autonomous City of Buenos Aires, Argentina.

b. Department of Surgery of Hospital de Pediatría "Prof. Dr. Juan P. Garrahan," Autonomous City of Buenos Aires, Argentina.

c. Laboratory: Department of Parasitology of Hospital de Pediatría "Prof. Dr. Juan P. Garrahan," Autonomous City of Buenos Aires, Argentina.

d. Laboratory:Department of Serology of Hospital dePediatría "Prof. Dr. Juan P. Garrahan," Autonomous City of Buenos Aires, Argentina.

E-mail address:

Griselda Berberian, M.D.: griselberberian@yahoo.com.ar

Funding: None.

Conflict of interest: None.

Received: 7-21-2016

Accepted: 12-5-2016

\section{INTRODUCTION}

Echinococcosis is a zoonotic parasitic disease caused by cestodes (tapeworms) of the genus Echinococcus. The only type of clinical relevance in the American continent is Echinococcus granulosus. This disease is caused by the larval stages, for which humans are accidental intermediate hosts. It is the most common cause of liver cysts worldwide, and one of the most neglected diseases $^{1}$ (Figure 1). In Argentina it is considered endemic, with more than 300 new cases every year. ${ }^{2,3}$

\section{OBJECTIVE}

To establish the epidemiology, clinical characteristics, treatment, and course of children with echinococcosis hospitalized at a tertiary care children's hospital outside the endemic area.

\section{POPULATION AND METHODS}

Retrospective review of medical records of children $\leq 18$ years old with an E. granulosus infection based on the international diagnostic criteria established by the World Health Organization (WHO) considering the epidemiology, clinical characteristics, images, anatomic pathology, serology, and parasitology of echinococcosis. ${ }^{4}$

The study period covered 20 years (May 1993-October 2013).

Outcome measures of interest included age, sex, place of origin, risk factors, clinical diagnosis, images, general lab tests, serological tests, treatment, and course.

Positive findings on epidemiology were defined as the presence of one or more risk factors for acquisition, such as origin from an endemic or rural area or having dogs. Serology was established using an enzyme-linked immunosorbent assay (ELISA) and confirmed using a Western blot for special situations. Imaging tests were selected based on the location and clinical presentation of echinococcosis.

Data were processed using the Epi-Info 6.0 software. Continuous outcome measures were reported as mean or median and range. 
Categorical outcome measures were expressed in number and percentage.

\section{RESULTS}

Forty-seven patients were included; their median age was 8 years old. This is a referral hospital, so patients come from different places: 43 patients (91\%) were from Argentina and 4 (9\%), from Bolivia. Within the Argentine region, places of origin corresponded to the province of Buenos Aires (20 patients, 47\%), Northeast (9 patients, 21\%), Northwest (7 patients, $16 \%$ ), and Patagonia (7 patients, $16 \%$ ).

Forty patients $(85 \%)$ had cysts located in only one parenchyma. Out of the 54 cyst locations, the liver and lungs were the most common ones; 45 patients (96\%) received medical/surgical treatment. Complications were observed in 13 patients $(28 \%)$. Pulmonary complications included bronchopleural fistula, pneumothorax, diaphragmatic paresis, atelectasis, and Staphylococcus aureus bacteremia. Complications related to brain cysts included intraoperative cyst rupture and hydrocephalus. In relation to patients who had complications from liver cysts, all had infections, including wall abscesses and secondary liver abscesses. Three patients $(6 \%)$ relapsed between 1 and 24 months later and required a new surgery (Table 1 ).

\section{DISCUSSION}

In the region of the Americas, the largest number of echinococcosis cases is reported in South America. There are different species depending on the geographic area, with at least 10 distinct genetic types of E. granulosus. ${ }^{5}$

In Argentina, it is distributed across the entire national territory, especially in rural areas for sheep and goat breeding in the Patagonia, Mesopotamia, Humid Pampa, Cuyo, and Northwest. ${ }^{6}$

Echinococcosis is a zoonotic disease, so epidemiological history is essential for diagnostic guidance. Its clinical spectrum ranges from an asymptomatic presentation to a severe form, but it is rarely fatal, depending on cyst location, size, characteristics, and the presence or absence of complications.

Echinococcosis mostly involves the liver (67$89 \%)$ and, less frequently, the lungs (10-25\%). It may occur in the spleen (1-3\%), peritoneum (3$5 \%)$, kidneys (2\%), brain (2\%) and, more rarely, the heart, bones, pancreas, and orbit. ${ }^{7-9}$ Atypical and complicated locations require to be managed in tertiary care hospitals.

Single cysts are observed in $60-80 \%$ of cases. In the series presented here, single cysts in only one parenchyma were the most common type; and the liver and lungs were the most common locations.

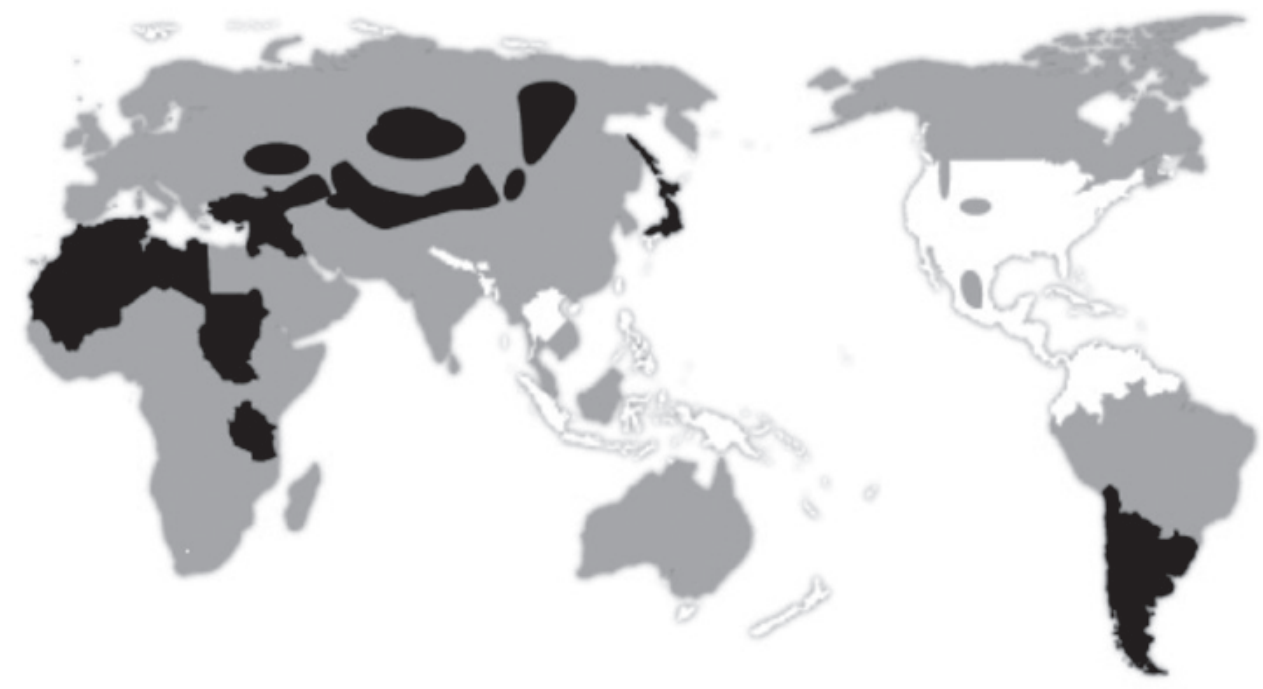

Echinococcosis hyperendemic regions 
The biased increased in pulmonary echinococcosis is related to the need of referral for specialized surgical treatment given that this is a tertiary care hospital, so it provides care for atypical or complicated forms requiring differential diagnoses and multidisciplinary management.

Diagnosis is based on epidemiological history, clinical characteristics, and additional imaging and lab tests, surgical findings, and pathological examination. Among additional tests, imaging studies to establish cyst location are essential for diagnostic guidance.

Initially, conventional X-rays are used to diagnose pulmonary echinococcosis, unlike the case of abdominal presentation, for which the imaging technique of choice for the WHO is ultrasonography. ${ }^{10}$ Other studies such as computed tomography scans or magnetic resonance imaging tests are used for specific surgical guidance and classification (Figures 2, 3, and 4).

Indirect hemagglutination and ELISA tests are used for serological diagnosis, with a sensitivity ranging from $50 \%$ to $98 \%$. Uncertain cases are serologically confirmed by Western blotting. False negative results are more common when cysts are located in the lungs compared to the liver, with a $50-60 \%$ and $85-98 \%$ sensitivity, respectively; false negative results are even higher in the case of multiple or broken cysts (90-100\%). A negative serology result does not rule out echinococcosis. New diagnostic methodologies, such as the Ag5 ELISA and the polymerase chain reaction (PCR), are under assessment to improve sensitivity. ${ }^{11}$ For this reason, the presence of a cystic mass in a patient with a history of exposure to dogs and sheep in areas where E. granulosus is endemic would validate the diagnosis of echinococcosis. However, a differential diagnosis should always be done to rule out other cystic conditions, such as benign cysts, tuberculosis, fungal infections, abscesses or tumors. A chest X-ray should be done in all patients with hepatic echinococcosis to rule out its presence in the lungs, and their family should also be examined for parasitic diseases.

Treatment is provided based on the national and international WHO consensuses. ${ }^{2,4}$ Most

TABLE 1. Result of epidemiological and clinical characteristics, diagnosis, treatment, and course. N: 47 patients

\begin{tabular}{lll}
\hline Outcome measures & Number (n) & Percentage (\%) \\
Age at diagnosis (median years old) & 8 (range: 3-17) & 51 \\
Male patients & 24 & 98 \\
Positive epidemiological findings & 46 & 62 \\
$\quad$ Living in a rural area & 29 & \\
Time until diagnosis (median months) & 3 (range: 1-32) & 44 \\
LOCATION: & $\mathbf{5 4}$ & 39 \\
$\quad$ Lungs & 24 & 11 \\
$\quad$ Liver & 21 & 2 \\
Brain & 6 & 2 \\
$\quad$ Eyes & 1 & 2 \\
$\quad$ Ridneys & 1 & 85 \\
One parenchyma involved & 1 & 30 \\
Multiple cysts (one or more parenchymas) & 40 & 15 \\
Cysts in multiple organs & 14 & 23 \\
Eosinophilia (> 500/mm $\left.{ }^{3}\right)$ & 7 & 45 \\
Positive serology (ELISA) & 11 & 96 \\
Medical/surgical treatment & 21 & 91 \\
Open surgery & 45 & 7 \\
Laparoscopy & 41 & 2 \\
PAIR & 3 & $\mathbf{2 8}$ \\
Complications & 1 & 62 \\
$\quad$ Lungs & 13 & 23 \\
Liver & 8 & 15 \\
Relapse & 3 & 6 \\
\hline
\end{tabular}

PAIR: puncture, aspiration, injection, reaspiration; ELISA: enzyme-linked immunosorbent assay. 
guidelines are based on expert opinions because no good-quality evidence comparative studies have been conducted so far. Until 1980, surgery was the only treatment option; however, antiparasitic drugs and new percutaneous methods have replaced conventional surgical excision.

Treatment may be summarized into 4 basic modalities: conventional surgery, percutaneous techniques, antiparasitic drugs, and nonintervention (watch and wait). Surgery depends on the clinical course, location, size and characteristics of the cysts and the surgeon's experience. ${ }^{3}$ The most commonly recommended surgical techniques include open surgery (radical or conservative) and laparoscopy. Percutaneous techniques include puncture, aspiration, injection,

FIGURE 2. Lung location

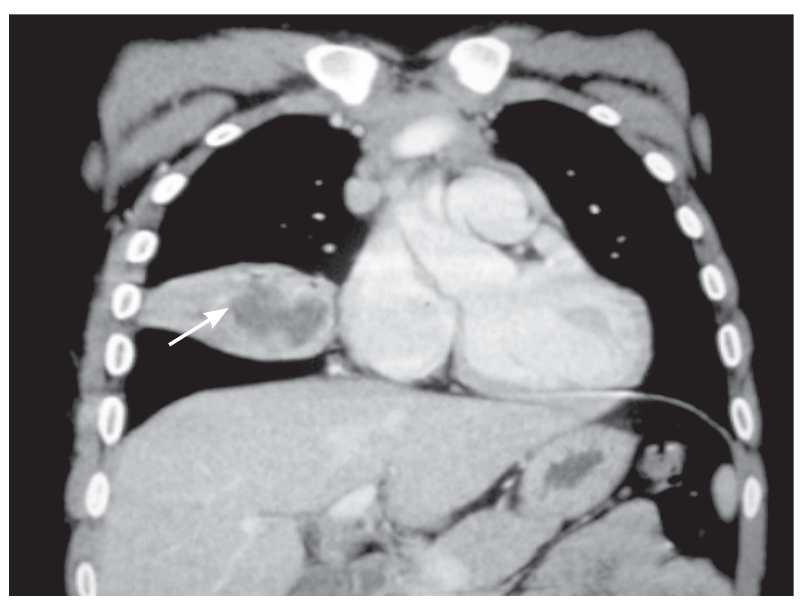

Computed tomography scan of the chest. reaspiration (PAIR). Conventional surgery is the main choice for symptomatic patients and those with large cysts, overinfected cysts, or cysts located in the brain, lungs or kidneys; in this case, mortality rate ranges between $0.5 \%$ and $4 \%$. The PAIR technique is a minimally invasive method used for cysts especially located in the liver and other locations, such as the abdomen, when these cannot be accessed through conventional techniques. It is contraindicated for superficial liver cysts and for inactive or calcified cysts, or in the case of bile duct communication and lung cysts. ${ }^{12}$ It has been estimated that the risk for postpuncture anaphylactic shock is $0.047 \%$, based on 16 cases suffering from this complication out of 4209 cyst punctures. ${ }^{13}$

Antiparasitic drugs are recommended for all cases as an adjuvant to surgery, or as monotherapy for asymptomatic patients with small cysts or multiple cysts that cannot be treated and cured with surgery. ${ }^{14}$

Albendazole and mebendazole have shown to be effective for echinococcosis treatment; however, results with albendazole have been superior due to its pharmacokinetics, intestinal absorption, and cyst penetration. Albendazole is the treatment of choice for continuous therapy starting one month prior to surgery up to 3-6 months after the procedure. The addition of praziquantel is recommended for surgical accidents with broken cysts or for severe cases to prevent secondary dissemination. ${ }^{15}$ The frequency and type of post-surgical complications range between $8.5 \%$ and $22 \%$, and depend especially on the location (complications are more common in the case of lung cysts).

In this series, 13 patients $(28 \%)$ had complications; most commonly among patients
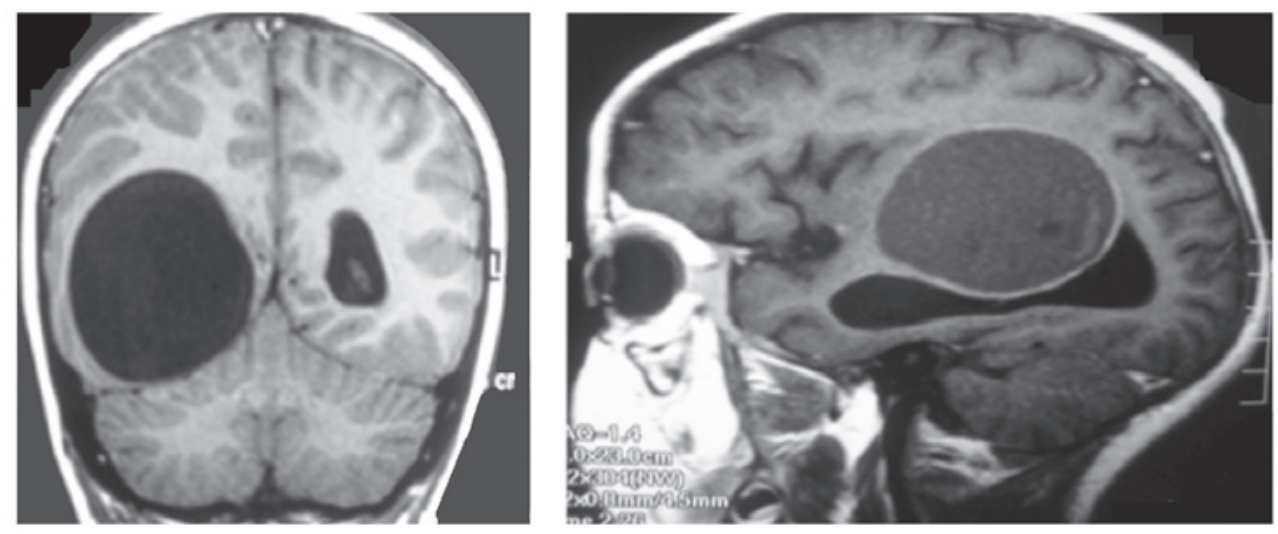

Magnetic resonance imaging of the brain. 
with lung cysts because this is a referral hospital. No patient died as a result of this disease.

In relation to prevention strategies, mathematical models have shown that the most effective intervention is a combination of cattle immunization and periodic dog deworming. This way, echinococcosis reduction is achieved among intermediate and definitive hosts. This should be combined with health education to prevent home slaughter of livestock and feeding dogs with viscera.

\section{CONCLUSION}

Echinococcosis should be considered in the diagnosis of children with cystic lesions, especially when cysts are located in the liver and/ or lungs. The management of this disease requires multidisciplinary work, and the surgical approach is a priority.

FiguRe 4. Orbital location

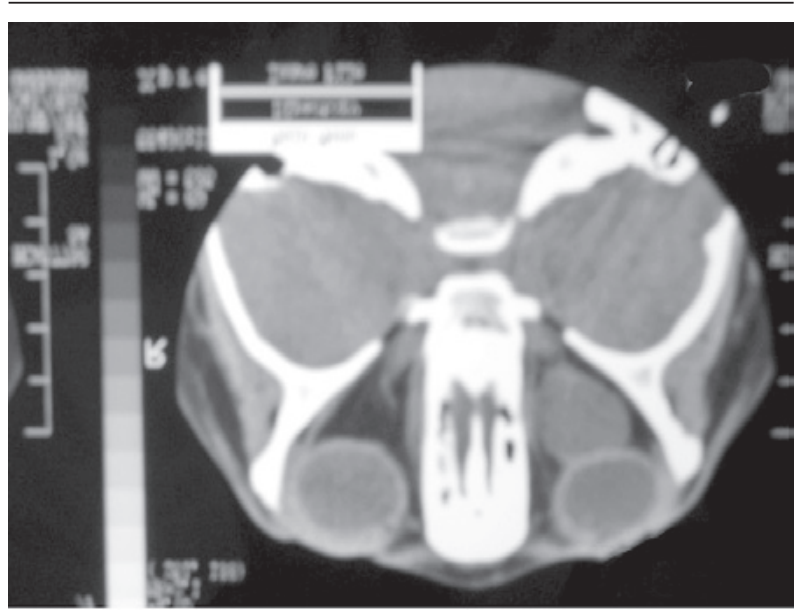

Computed tomography scan of the orbit and facial bones.

\section{REFERENCES}

1. Craig P Budka CM, Schantz PM, Li T, et al. Human echinococcosis: a neglected disease? Trop Med Health 2007;35(4):283-92.

2. Enfermedades infecciosas: Hidatidosis. Guías para el equipo de Salud. Buenos Aires: Ministerio de Salud de la República Argentina; 2012. [Accessed on: December 12 ${ }^{\text {th }}$, 2016]. Available at: http://www.msal.gob.ar/images/ stories / epidemiologia/pdf/guia-medica-hidatidosis.pdf.

3. Hidatidosis. Casos acumulados hasta la 43a semana epidemiológica. País Argentina por provincia. Años 20152016. Boletín Integrado de Vigilancia 2016;338(SE48):82.

4. BrunettiE,Kern P, Vuitton DA, Writing Panel for the WHOIWGE. Expert consensus for the diagnosis and treatment of cystic and alveolar echinococcosis in humans. Acta Trop 2010;114(1):1-16.

5. Moro P, Schantz P. Echinococcosis: a review. Int J Infect Dis 2009;13(2):125-33.

6. Consenso sobre enfermedades infecciosas regionales en la Argentina. Recomendaciones de la Sociedad Argentina de Pediatría-Comité Nacional de Infectología. 2012-13. [Accessed on: December 12 ${ }^{\text {th }}, 2016$. Available at: http:/ / sap.org.ar/docs/profesionales/ consensos/consenso_ patologia_regional.pdf.

7. Schaefer JW, Khan MY. Echinococcosis (Hydatid Disease): Lessons from experience with 59 patients. Rev Infect Dis 1991;13(2):243-7.

8. Moscatelli G, Abraham Z, Moroni S, Martínez Iriart E, et al. Hidatidosis pulmonar. Arch Argent Pediatr 2012;110(3): 265-6.

9. Tapia O, Vidal A, Antonio L. Hidatidosis cerebral: aspectos clínicos eimagenológicos. Reporte de 4 casos. Rev Med Chile 2012;140(3):358-63.

10. World Health Organization. International classification of ultrasound images in cystic echinococcosis for application in clinical and field epidemiological settings. Acta Trop 2003;85(2):253-61.

11. Paganozzi D, Addis MF, Biosa G, Roggio A, et al. Diagnostic Accuracy of Antigen 5-Based ELISAs for Human Cystic Echinococcosis. PLoS Negl Trop Dis 2016;10(3):e0004585.

12. Brunetti E, Junghanss T. Update on cystic Hydatid disease. Curr Opin Infect Dis 2009;22(5):497-502.

13. Smego RA Jr, Bhatti S, Khalij A, Beg MA. Percutaneous aspiration-injection-reaspiration drainage plus albendazole or mebendazole for hepatic cystic echinococcosis: a metaanalysis. Clin Infect Dis 2003;37(8):1073-83.

14. Junghanss $\mathrm{T}$, da Silva A, Horton J, Chiodini PL, et al. Clinical management of cysic echinococcosis: state of the art, problems, and perspectives. Am J Trop Med Hyg 2008;79(3):301-11.

15. Arif SH, Shams-Ul-Bari, Wani NA, Zargar SA, et al. Albendazole as an adjuvant to the standard surgical management of hydatid cyst liver. Int J Surg 2008;6(6): $448-51$. 